\title{
Neurofunctional correlates of expressed vocal affect in social phobia
}

\author{
Petri Laukka • Fredrik Åhs • Tomas Furmark • \\ Mats Fredrikson
}

Published online: 21 April 2011

(C) Psychonomic Society, Inc. 2011

\begin{abstract}
We investigated the neural correlates of expressed vocal affect in patients with social phobia. A group of 36 patients performed an anxiogenic public-speaking task while regional cerebral blood flow (rCBF) was assessed using oxygen-15 positron emission tomography. The patients' speech was recorded and content masked using low-pass filtering (which obscures linguistic content but preserves nonverbal affective cues). The content-masked speech samples were then evaluated with regard to their level of vocally expressed nervousness. We hypothesized that activity in prefrontal and subcortical brain areas previously implicated in emotion regulation would be associated with the degree of expressed vocal affect. Regression analyses accordingly revealed significant negative correlations between expressed vocal affect and $\mathrm{rCBF}$ in inferior frontal gyrus, putamen, and hippocampus. Further, functional connectivity was revealed between inferior frontal gyrus and (a) anterior cingulate cortex and (b) amygdala and basal ganglia. We suggest that brain areas important for emotion regulation may also form part of a network associated with the modulation of affective prosody in social phobia.
\end{abstract}

Keywords Anxiety PET P Prefrontal cortex · Social phobia Vocal affect expression

P. Laukka $(\bowtie)$

Department of Psychology, Stockholm University, 10691 Stockholm, Sweden

e-mail: petri.laukka@psychology.su.se

F. Åhs

Center for Cognitive Neuroscience, Duke University,

Durham, North Carolina

T. Furmark $\cdot$ M. Fredrikson

Department of Psychology, Uppsala University,

Uppsala, Sweden
Whenever people communicate through speech, they always convey affective expression nonverbally through their tone of voice (i.e., affective prosody) apart from the meaning communicated by their words (Juslin \& Laukka, 2003; Laukka, 2008; Russell, Bachorowski, \& FernándezDols, 2003; Scherer, 2003). People are also very adept at regulating their expressions, and may willfully conceal or strategically pose their vocal expressions in everyday life (Cowie \& Cornelius, 2003; Scherer, 1989). The modulation of affective prosody thus may well be one of the most common emotion regulation strategies in everyday human interactions, but the neural mechanisms underlying this phenomenon have received little attention. In the present study, we explored the neural correlates of expressed vocal affect in a real emotion-eliciting situation using data from a large ongoing study of social phobia treatment (see Furmark, Appel, Michelgård, Wahlstedt, Åhs, Zancan, et al., 2005).

In short, we had social phobia patients perform an anxiety-provoking public speech task while their regional cerebral blood flow (rCBF) was assessed using positron emission tomography. The patients' speech was recorded and content-masked using low-pass filtering (which obscures the linguistic content but preserves nonverbal affective cues) prior to being evaluated by a group of listeners with regard to the level of vocally expressed nervousness. Then, in order to map the functional neuroanatomy of expressed vocal affect, the correlations between $\mathrm{rCBF}$ in predefined regions of interest (ROIs) and the level of vocally expressed nervousness were calculated. We hypothesized that $\mathrm{rCBF}$ in prefrontal and subcortical areas previously implicated in emotion regulation and vocal control would be correlated with the amount of nervousness expressed nonverbally through the voice.

The study of emotion regulation has recently appeared as one of the main enterprises in the cognitive and affective 
neurosciences (see, e.g., Lieberman, 2007). Emotions may be conceived of as brief and intense reactions to goal-relevant changes in the environment, with the emotional reaction involving several components, including cognitive appraisals, subjective feelings, physiological arousal, expression, and action tendencies (e.g., Scherer, 2000). Consequently the regulation of emotion may take many forms, and the "effects of emotion regulation can be observed across all modalities of emotional responding, including behavior, physiology, thoughts and feelings" (Koole, 2009, p. 7; see also Gross, 1998). Most neuroimaging research has focused on the voluntary behavioral inhibition of reactions to emotional stimuli (suppression), or on the cognitive reinterpretation of evocative stimuli to reduce negative affect (reappraisal). The prefrontal cortex (PFC) has been appointed a key player in emotion regulation, as indicated by several reviews (e.g., Davidson, 2002; Ochsner \& Gross, 2005; Phillips, Drevets, Rauch, \& Lane, 2003; Phillips, Ladouceur, \& Drevets, 2008; Quirk \& Beer, 2006). Among many examples, suppression of emotional expressions while viewing film clips has been associated with activity within bilateral dorsomedial PFC (DMPFC), right dorsolateral PFC (DLPFC), and left ventrolateral PFC (VLPFC) (Goldin, McRae, Ramel, \& Gross, 2008; Gross \& Levenson, 1997; Hagemann, Levenson, \& Gross, 2006). Reappraisal, in turn, has also been associated with greater activity in left lateral and medial PFC during, for example, reinterpretation of negative pictures so that they no longer elicit negative subjective emotional responses (Ochsner, Bunge, Gross, \& Gabrieli, 2002). Increased activation of orbitofrontal cortex (OFC) and rostral anterior cingulate cortex (ACC) has further been associated with attenuated amygdala reactivity during reappraisal-based negative affect down-regulation (Banks, Eddy, Angstadt, Nathan, \& Phan, 2007; Ochsner et al., 2002; Urry, van Reekum, Johnstone, Kalin, Thurow, Schaefer, et al., 2006). Finally, Wager, Davidson, Hughes, Lindquist, \& Ochsner, (2008) showed that VLPFC was involved in both the generation and regulation of emotion through different subcortical pathways, suggesting a general role for this region in appraisal processes.

In a synthesis of the neuroimaging literature on emotion regulation, Ochsner and Gross (2007) proposed that the lateral and medial PFC, together with the ACC, form a topdown appraisal system that controls bottom-up appraisals generated by subcortical regions such as the amygdala and the basal ganglia/striatum (see also Kober, Barrett, Joseph, Bliss-Moreau, Lindquist, \& Wager, 2008). Phillips et al. (2008) likewise suggested that lateral and medial PFC systems are indicated as participating in emotion regulation. More specifically, they argued that the lateral system (including, e.g., DLPFC and VLPFC) is mainly involved in voluntary subprocesses, whereas the medial system (including, e.g., OFC, rostral and ventral ACC, and DMPFC) may instead subserve automatic subprocesses. Animal lesion, clinical neuropsychological, and psychophysiological studies have also suggested that the PFC plays a critical role in the top-down regulation of activity of emotion-related limbic areas including the amygdala (for reviews, see Davidson, Fox, \& Kalin, 2007; Lane \& McRae, 2004).

Emotion regulation plays a central role in both the etiology and the maintenance of clinically relevant levels of psychopathology (see, e.g., Kring \& Bachorowski, 1999), and social phobia (social anxiety disorder) is characterized by excessive fear of scrutiny by others and an excessive drive to suppress and inhibit anxious behaviors in social situations (e.g., Campbell-Sills, Barlow, Brown, \& Hofmann, 2006; Kashdan \& Steger, 2006; Spokas, Luterek, \& Heimberg, 2009). Prior neuroimaging studies suggested that social phobia is characterized by emotion dysregulation and hyperactivation in limbic and paralimbic regions such as the amygdala, reflecting overactivation of a core fear system (for reviews, see Engel, Bandelow, Gruber, \& Wedekind, 2009; Etkin \& Wager, 2007; Freitas-Ferrari, Hallak, Trzesniak, Filho, Machado-de-Sousa, Chagas, et al., 2010). Patients with anxiety disorders have also shown abnormalities in both spontaneous and instructed emotion regulation, with altered functioning of medial and lateral PFC (Campbell-Sills, Simmons, Lovero, Rochlin, Paulus, \& Stein, 2011; Goldin, Manber, Hakimi, Canli, \& Gross, 2009) and ACC (Etkin, Prater, Hoeft, Menon, \& Schatzberg, 2010) as compared with healthy controls. No prior studies have investigated the neural mechanisms involved in expressed vocal affect in social phobia, but a better understanding of this topic seems to be a pertinent issue with implications for patients' emotional and social functioning.

Neuroimaging research on vocal affect expression has mainly been concerned with the neural correlates of affective prosody recognition (e.g., Ethofer, Van De Ville, Scherer, \& Vuilleumier, 2010; Leitman, Wolf, Ragland, Laukka, Loughead, Valdez, et al., 2010; Schirmer \& Kotz, 2006), and very few studies have investigated the production or modulation of affective prosody. Based on evidence from clinical lesion studies, Ross (1981) suggested that disorders of spontaneous affective prosody production resulted from damage to right inferior frontal lobe damage, in parallel to the speech production deficits associated with left Broca's area lesions, but subsequent studies have failed to find consistent support for his model of right hemisphere organization (see Baum \& Pell, 1999). Jürgens (2002, 2009), on the basis of his research in the squirrel monkey, has proposed that the ACC is involved in the voluntary initiation of vocal behavior (including affective prosody). Of special interest to the present study are findings that bilateral lesions to the ACC prevent the volitional initiation of vocal responses in emotionally relevant situations in monkeys (e.g., Aitken, 1981; MacLean \& Newman, 1988). 
Evidence from humans is sparser, but a case study reported that a lesion in the ACC produced a permanent deficit in affective prosody production. However, the deficit was limited to volitional expressions (i.e., the patient was no longer able to express distinct emotions whenever he wanted to do so), whereas unvolitional expressions (i.e., laughing and crying) were spared (Jürgens \& von Cramon, 1982; von Cramon \& Jürgens, 1983). Also, Barrett, Pike, and Paus (2004) reported that the ACC may modulate the prosodic characteristics of speech during experimentally induced affective states in humans. More specifically, for a group of participants who completed a speech task both before and after a sadness induction procedure, the affectrelated changes in pitch variation were found to correlate with greater activity in dorsal (supracallosal) areas of ACC.

The above review of relevant studies shows that prefrontal brain areas have consistently been implicated in both emotion regulation (e.g., Ochsner \& Gross, 2007; Phillips et al., 2008) and vocal control (ACC; see Jürgens, 2002), and we therefore chose these areas as our a priori ROIs in the present study. Specifically, we hypothesized that activity in lateral and medial PFC would be negatively correlated with the degree of vocally expressed nervousness, if these areas are involved in the down-regulation of affective prosody. Because previous studies suggested an interaction between cortical and subcortical areas in emotion regulation, we also included subcortical areas (i.e., amygdala, hippocampus, and the basal ganglia; see Phillips et al., 2008) in our analyses, to explore whether these areas may show any association with expressed vocal affect. Finally, we investigated the functional connectivity between prefrontal and subcortical areas, as related to expressed vocal affect. In this regard, we expected to find functional connectivity if the brain areas in question formed part of a network associated with affective prosody.

\section{Method}

\section{Participants}

A group of 36 participants ( 24 female, 12 male; mean age $=$ 33 years) were included in the present study. Data were originally collected in the framework of a large ongoing project concerning pharmacological treatment for social phobia conducted at Uppsala University, wherein patients with social phobia performed an anxiogenic public-speaking task while their regional cerebral blood flow and speech were recorded (e.g., Furmark et al., 2005; Furmark, Tillfors, Marteinsdottir, Fischer, Pissiota, Långström, et al., 2002). All participants reported having had symptoms during a long period and met the DSM-IV criteria for social phobia (social anxiety disorder), as revealed by structured clinical inter- views (Structured Clinical Interview for DSM-IV; First, Gibbon, Spitzer, \& Williams, 1998). Of our patient group, 27 met the criteria for generalized social phobia, whereas 9 had the nongeneralized subtype. No participant had received treatment for social anxiety in the past 6 months or had current serious or dominant psychiatric disorders (e.g., depression) other than social phobia. Importantly, no patient used psychoactive drugs or any prescribed medication chronically (for a more thorough description of the screening procedure, see Furmark et al., 2005). All patients were right handed. The studies were approved by the Uppsala University Medical Faculty Ethical Review Board, the Swedish Medical Products Agency, and the local radiation ethics committee. In the present study, we used data recorded from the baseline sessions only (i.e., before any treatment was administered). Written informed consent was obtained from all participants.

For the purposes of the present study, we wanted to have a sample of participants who all reported high levels of state anxiety, but at the same differed widely regarding their levels of expressed vocal affect. The participants' levels of state anxiety and vocally expressed affect had been assessed in a previous study (Laukka et al., 2008), and in the present study we included patients who responded with high anxiety (at or above the median response) to the public-speaking task from the sample used in that study. Below, we describe how expressed vocal affect and state anxiety were assessed.

Assessment of participants' levels of expressed vocal affect In a previous study, 16 listeners had judged the level of nervousness ("in your opinion, how nervous does the speaker sound?") of the speech stimuli on a scale from 0 (not nervous at all) to 10 (very nervous) (Laukka et al., 2008). To avoid misunderstandings, "nervousness" was carefully explained to the listeners as feelings of apprehension, fear, nervousness, tension, and worry. The internal consistency of ratings among the judges (as measured by Cronbach's alpha: $\alpha=.91$ ) and the interrater reliability (as measured by the average measure intraclass correlation using the Spearman-Brown formula: $R=.81$ ) were good for the nervousness scale. The mean level of vocally expressed nervousness in our sample was 5.44 (SD = $1.15)$, and the sample included both patients who did and did not sound nervous (the nervousness ratings for the individual patients ranged from 3.31 to 8.13 ).

The speech stimuli consisted of the first $10 \mathrm{~s}$ of speech recorded at the public-speaking task. A wide range of studies has shown that $10 \mathrm{~s}$ is long enough to allow accurate social and interpersonal perception from expressive behavior, including nonverbal aspects of speech (Ambady \& Rosenthal, 1992). Before being entered into the listening test, the speech stimuli were content masked by low-pass filtering, where all frequencies above $500 \mathrm{~Hz}$ were removed using the Praat 
software (Boersma \& Weenink, 2007). This procedure eliminates phonetic information and renders the speech unintelligible and muffled sounding, while largely preserving affective information transmitted by pitch, voice intensity, and temporal aspects of the speech (e.g., Scherer, Koivumaki, \& Rosenthal, 1972; van Bezooijen \& Boves, 1986). The filtering procedure forced the listeners to focus on the nonverbal content, as opposed to the linguistic content, of the speech, and also preserved the anonymity of the speakers. The listening tests were conducted individually using custom computer software, and the judges listened to the stimuli presented in random order through headphones. We refer the reader to Laukka et al. (2008) for a full description of the recording and listening test procedures.

A detailed report of the associations between listeners' nervousness ratings and the acoustic characteristics of the speech samples is reported in Laukka et al. (2008). In brief, nervousness was associated with nonverbal cues such as high pitch, low voice intensity, and a large proportion of silent pauses, but not with the number of words produced. In our sample, only mean pitch (i.e., fundamental frequency) was significantly correlated with nervousness ratings (Pearson $r=.46, p<.01$ ), which suggests that the listeners utilized nonverbal affective information transmitted by pitch to make their inferences of vocally expressed affect.

Assessment of participants' self-reported anxiety Selfreported anxiety was assessed by using the State scale of the Spielberger State-Trait Anxiety Inventory (STAIS-S; Spielberger, Gorsuch, Lushene, Vagg, \& Jacobs, 1983). The STAI-S scale consists of 20 self-descriptive items that measure how the participant feels at a particular moment (e.g., "I feel nervous") rated on a scale from 1 to 4 (from not at all to somewhat, moderately so, and very much so, respectively). The essential qualities evaluated by the STAI-S scale are feelings of apprehension, tension, nervousness, and worry. The STAI-S scores can range from 20 to 80 , and higher scores indicate higher levels of state anxiety. All participants filled in the STAI-S scale directly after they had performed their speech in order to estimate retrospectively how anxious they had felt during the publicspeaking task. In the present study, we included only patients who had reported high anxiety (STAI-S scores at or above the median; median $=64$ ) in response to the publicspeaking task from the original selection described in Laukka et al. (2008). The STAI-S scores for our sample $(M=69.9, S D=4.09$, range $=64-80)$ suggested that the anxiety manipulation resulted in relatively intense anxiety. Further, the STAI-S scores and the vocal nervousness ratings were not significantly correlated (Pearson $r=-.06$, $p=.72$ ) in our participant sample.

\section{Procedure}

The participants performed a public-speaking task while lying in a PET scanner. About $20 \mathrm{~min}$ before the scanning began, they were instructed to prepare a speech. The topic of the speech was to tell the audience about a recent vacation/travel experience and was the same for all participants. When the scanning began, the patients were asked to start speaking and to continue for approximately 2 min until they received instructions to stop. The speech was performed before an audience of six to eight persons who silently observed the patient's performance, and the patients were further instructed to watch the audience in order to elicit observational anxiety.

We chose a public-speaking task as our method of anxiety provocation because speaking in public is the most prevalent social fear, both in individuals with social phobia and in the community at large (Furmark et al., 1999; Kessler, Stein, \& Berglund, 1998). Also, we have previously shown that individuals with social phobia experience relatively high anxiety in this type of publicspeaking task, as indicated by self-reports (e.g., Tillfors, Furmark, Marteinsdottir, Fischer, Pissiota, Långström et al., 2001). In addition, cerebral blood flow was also increased in areas previously associated with heightened anxiety (e.g., Furmark et al., 2002).

\section{PET assessments}

All participants were examined with a 32-ring ECAT EXACT HR + PET scanner (Siemens/CTI, Knoxville, Tennessee). The scanner enabled acquisition of 63 contiguous planes of data with a distance of $2.46 \mathrm{~mm}$, resulting in a total axial field of view of $155 \mathrm{~mm}$. Participants were positioned in the scanner with the head gently fixated, and a venous catheter for tracer injections was inserted. Patients were instructed to prepare their speech about $20 \mathrm{~min}$ before the initial emission scan. A 10-min transmission scan was performed using three retractable germanium $\left({ }^{68} \mathrm{Ge}\right)$ rotating line sources. The ${ }^{15} \mathrm{O}$-water tracer, approximately $10 \mathrm{MBq} / \mathrm{kg}$ body weight, was thereafter injected intravenously. The emission scan started automatically in three-dimensional mode when the bolus reached the brain $(50,000$ counts/s) and consisted of three 30-s frames. Immediately following the tracer injection, patients were asked to start speaking and to continue until they received instructions to stop.

Emission scans were reconstructed with a filter back projection using an 8-mm Hanning filter, resulting in a spatial resolution of about $5 \mathrm{~mm}$ in the field of view. The matrix included $128 \times 128$ pixels. Data were corrected for photon attenuation, decay, scattered radiation, and random coincidences. After reconstruction, a summation image of the three frames was made in order to obtain a 
better statistical reference for realignment and subsequent analyses.

Participants fasted $3 \mathrm{~h}$ and refrained from tobacco, alcohol, and caffeine for $12 \mathrm{~h}$ before the PET investigations.

\section{Neuroimaging analyses}

The PET images were realigned to correct for different positions between scans and were normalized to the Montreal Neurological Institute's (MNI) stereotaxic template (ICBM 152), using the Statistical Parametric Mapping (SPM2) software (Wellcome Department of Cognitive Neurology, London, U.K.). Images were then smoothed using a 12-mm Gaussian kernel. The covariation between PET data and vocally expressed nervousness was statistically evaluated using regression analyses defined in SPM with $\mathrm{rCBF}$ data fitted to the general linear model (Friston et al., 1994). To control for individual differences in selfreported state anxiety, the patients' STAI-S scores were included as covariates in the regression models. Differences in global blood flow were corrected for using the proportional scaling method within SPM2. Functional connectivity analyses were performed separately for left and right inferior frontal gyrus (IFG) by extracting the means of the activation clusters and entering these values as regressors in the design matrix. Simulations and analysis of neuroimaging data have shown that family-wise error correction might be too conservative under many circumstances (Nichols \& Hayasaka, 2003), and consequently we used a false discovery rate to correct for false positives in all analyses (Genovese, Lazar, \& Nichols, 2002).

Brain locations are described as $x, y, z$ coordinates in Talairach space, which we obtained by a nonlinear transformation of the MNI coordinates (http://imaging. mrc-cbu.cam.ac.uk/imaging/MniTalairach). Anatomical localization was supported by visual searches in the Talairach atlas (Talairach \& Tournoux, 1988) and the Internet-based Talairach Daemon (Lancaster, Woldorff, Parsons, Liotti, Freitas, Rainey, et al., 2000). In line with our a priori hypothesis, we defined the lateral and medial prefrontal cortices, together with subcortical areas (basal ganglia/ striatum and amygdala/hippocampal areas), as the ROIs for our analyses. ROIs were created in MNI space using the AAL library in the WFU Pickatlas software (Maldjian, Laurienti, Kraft, \& Burdette, 2003). The lateral PFC ROI was defined as the combination of the inferior orbital, inferior operculum, and inferior triangularis (Brodmann areas [BAs] 9, 44, 45, 46, and 47). The medial PFC/ACC ROI was also selected from the AAL library and included BAs 24, 25, and 32. The amygdala/hippocampus ROI included the amygdala and the hippocampus, and the basal ganglia ROI included the caudate, pallidum, and putamen, all from the AAL library in WFU Pickatlas. The volumes of our ROIs were $42,060 \mathrm{~mm}^{3}$ for the lateral PFC ROI; $21,700 \mathrm{~mm}^{3}$ for the medial PFC/ACC ROI; $29,552 \mathrm{~mm}^{3}$ for the basal ganglia/striatum ROI; and $18,616 \mathrm{~mm}^{3}$ for the amygdala/hippocampus ROI. Because there were no previous studies on expressed vocal affect, we deliberately chose the ROIs to be large enough to cover areas previously implicated in emotion regulation. Even though previous reports consistently implicated, for example, medial and lateral areas of the PFC in these processes, the exact location of the activations differed somewhat between studies. The definition of our ROIs therefore was a balance between covering enough brain area not to exclude vital areas and restricting the volume enough to give strong statistical power. In addition to the ROI analyses, we also performed exploratory whole-brain regression analyses between $\mathrm{rCBF}$ and expressed vocal affect.

\section{Results}

Covariation between expressed vocal affect and $\mathrm{rCBF}$

For our predefined ROIs in lateral and medial PFC, we found significant negative correlations - controlling for the patients' level of self-reported state anxiety-between $\mathrm{rCBF}$ and vocally expressed nervousness in lateral PFC. The local maxima were localized bilaterally in the IFG (BA 47), see Table 1 and Fig. 1. No significant correlations were found for the medial PFC ROI. These results suggest that patients who expressed little nervousness nonverbally had comparatively higher rCBF in IFG than did patients who expressed much nervousness nonverbally. For the subcortical ROIs, we further found significant negative correlations between vocally expressed nervousness and $\mathrm{rCBF}$ in right putamen and right hippocampus (see Table 1).

We also conducted an exploratory whole-brain regression analysis between $\mathrm{rCBF}$ and vocally expressed nervousness (also shown in Table 1), and this analysis revealed several additional brain regions in which $\mathrm{rCBF}$ covaried negatively with expressed vocal affect. Besides the areas in right IFG and hippocampus also implicated in the ROI analyses, additional areas revealed in the exploratory analyses included a cluster spanning the superior temporal and inferior frontal gyri, as well as clusters in superior frontal gyrus, precentral gyrus, precuneus, and cerebellum. All local maxima in the exploratory analyses, except for the cerebellum, were right lateralized.

Functional connectivity

We investigated the functional connectivity between voxels in the IFG that covaried with vocally expressed nervousness and the ACC and our subcortical ROIs. Functional 
Table 1 Regions in which regional cerebral blood flow was negatively correlated with expressed vocal affect, controlling for self-reported anxiety, along with $z$ scores representing local maxima and their associated Talairach coordinates, number of voxels/cluster, and voxelwise $p$ values corrected for multiple comparisons (false discovery rate correction)

\begin{tabular}{|c|c|c|c|c|c|c|}
\hline \multirow[b]{2}{*}{ Region } & \multirow[b]{2}{*}{$z$ score } & \multicolumn{3}{|c|}{ Coordinate } & \multirow[t]{2}{*}{ Voxels in cluster } & \multirow[t]{2}{*}{ Voxel $p$ value } \\
\hline & & $x$ & $y$ & $z$ & & \\
\hline \multicolumn{7}{|l|}{ Lateral PFC ROI } \\
\hline \multirow{3}{*}{$\begin{array}{l}\text { Right inferior frontal and superior temporal gyri } \\
\text { (BA } 47 \& 38 \text { ) }\end{array}$} & 4.42 & 53 & 33 & -8 & 1,262 & .004 \\
\hline & 4.37 & 44 & 21 & -16 & & 0.004 \\
\hline & 3.37 & 46 & 21 & -1 & & 0.009 \\
\hline \multirow[t]{2}{*}{ Left inferior frontal gyrus (BA 47) } & 3.35 & -36 & 29 & -6 & 396 & .009 \\
\hline & 3.29 & -38 & 26 & -16 & & 0.011 \\
\hline \multicolumn{7}{|l|}{ Medial PFC ROI } \\
\hline Right anterior cingulate cortex & 3.10 & 6 & 43 & 7 & 61 & .164 (n.s.) \\
\hline \multicolumn{7}{|l|}{ Basal ganglia ROI } \\
\hline Right putamen & 2.29 & 34 & 6 & -2 & 117 & .048 \\
\hline \multicolumn{7}{|l|}{ Amygdala/hippocampus ROI } \\
\hline Right hippocampus & 3.70 & 30 & -33 & -2 & 63 & .030 \\
\hline \multicolumn{7}{|c|}{ Exploratory (Whole-Brain) Analysis } \\
\hline Right superior temporal and inferior frontal gyri & 4.78 & 36 & 16 & -36 & 1,583 & .012 \\
\hline \multirow[t]{2}{*}{ (BA $38 \& 37$ ) } & 4.76 & 42 & 16 & -26 & & 0.012 \\
\hline & 4.42 & 53 & 33 & -8 & & 0.012 \\
\hline Left cerebellum (pyramis) & 3.77 & -22 & -77 & -33 & 151 & .033 \\
\hline Right precuneus (BA 31) & 3.73 & 6 & -47 & 34 & 68 & .035 \\
\hline Right hippocampus & 3.70 & 30 & -33 & -2 & 102 & .037 \\
\hline Right precentral gyrus (BA 4) & 3.69 & 42 & -19 & 38 & 94 & .038 \\
\hline Right superior frontal gyrus (BA 10) & 3.63 & 30 & 52 & -3 & 173 & .043 \\
\hline
\end{tabular}

connectivity analyses allow for the investigation of correlations between activity in different brain areas during task performance, and we expected to find functional connectivity if the brain areas in question were involved in a network associated with affective prosody. Voxels in the right and left IFG that showed maximum correlation with vocally expressed nervousness were chosen as the seed regions, and these values were entered as regressors in the design matrix. Functional connectivity was revealed ipsilaterally between both the left and right IFG and ACC, see Table 2 and Fig. 2. The local maximum in the right ACC was located in a rostral (perigenual) area, whereas the left ACC activation was located in a dorsal (supracallosal) area. For the subcortical ROIs, positive functional connectivity with IFG was revealed for several areas in the basal ganglia (putamen, pallidum, and caudate) and in limbic regions (amygdala and hippocampus; see Table 2).

\section{Discussion}

To summarize, we hypothesized that activity in prefrontal and subcortical brain territories previously implicated in emotion regulation and vocal control would also be associated with the degree of expressed vocal affect during an anxiogenic public-speaking task. We found that the level of nonverbally expressed nervousness was negatively correlated with $\mathrm{rCBF}$ in a-priori-defined ROIs in IFG, putamen, and hippocampus. Whole-brain correlational analyses further revealed additional brain areas that were associated with vocally expressed affect, including additional frontal as well as temporal and parietal areas. Finally, functional connectivity was revealed between IFG and frontal ACC, on the one hand, and amygdala, hippocampus, and the basal ganglia, on the other. These findings are discussed below.

Our finding that rCBF in IFG was correlated with vocally expressed affect is in line with previous research that has shown activation of lateral PFC in response to suppression of emotion expressions (e.g., Goldin et al., 2008; Gross \& Levenson, 1997; Hagemann et al., 2006; Johnstone, van Reekum, Urry, Kalin, \& Davidson, 2007). In the previous studies, regulation resulted in less intensively experienced affect, while LPFC at the same time showed enhanced activity. This suggests that LPFC may not be involved in the experience of emotion per se, but rather in the suppression of 
Fig. 1 Prefrontal regions in which regional cerebral blood flow (rCBF) was negatively correlated with expressed vocal affect. The results suggest that patients who expressed little nervousness had comparatively higher rCBF bilaterally in the inferior frontal gyrus (IFG) than did patients who expressed much nervousness. The functional images are superimposed on a standard magnetic resonance image, and the color bar represents $t$ scores
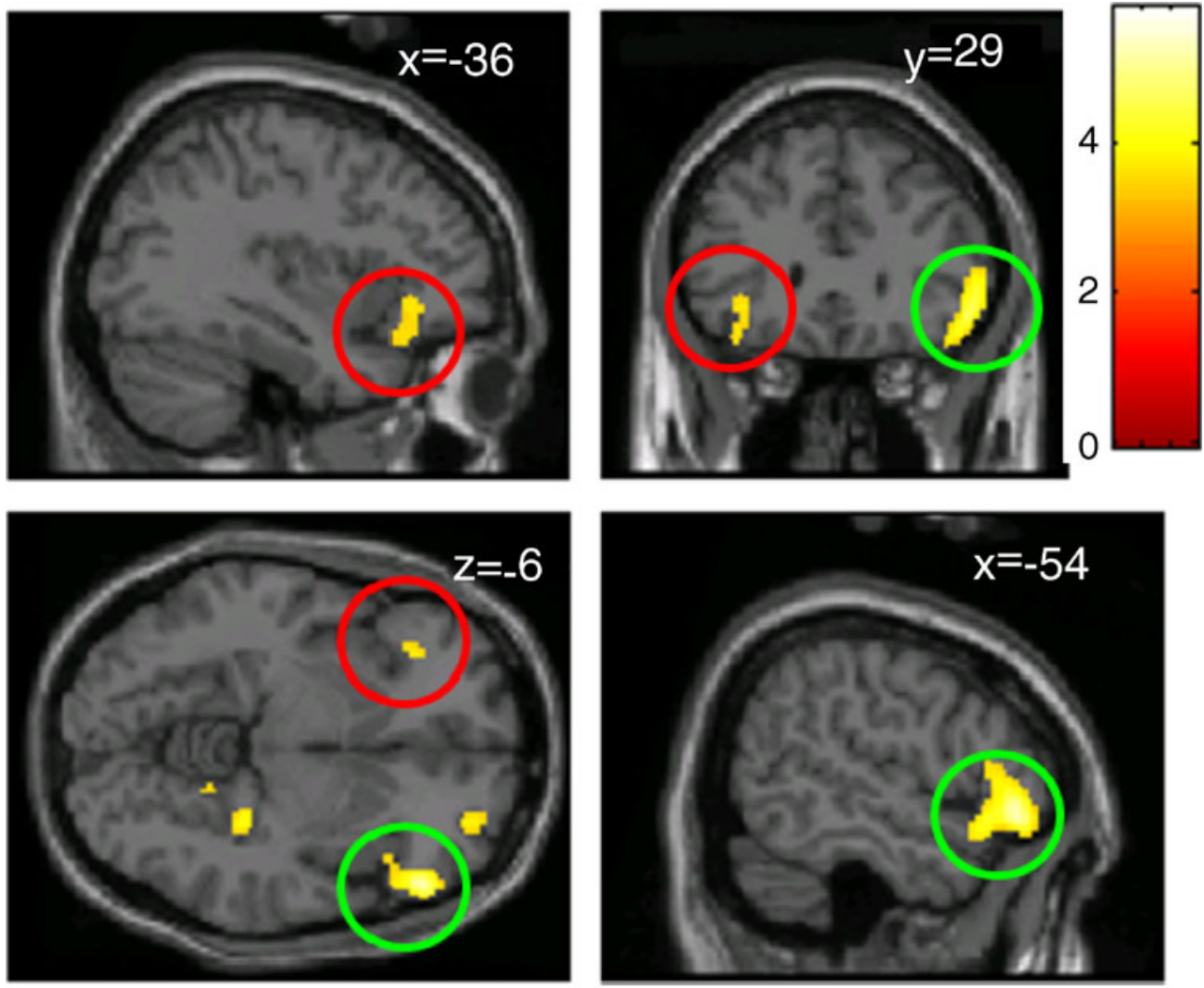

Left IFG

Right IFG other areas responsible for the emotional experience. In contrast to previous studies, in our study the participants who expressed less vocal affect did not experience less anxiety during the stressful speech task (as supported by the anxiety self-reports), which further suggests that LPFC (specifically, IFG) may be involved in the regulation of emotional behavior at a more general level.

Recent studies have shown that activity in IFG is associated with the deliberate production (acting) of both facial expressions (Lee, Josephs, Dolan, \& Critchley, 2006) and affective prosody (Aziz-Zadeh, Sheng, \& Gheytanchi, 2010). Our results were obtained with real, spontaneous vocal affect expressions rather that acted portrayals, but these findings are still relevant for our study, because regulation of genuine expressions entails that the expressions may be strategically posed as well as willfully suppressed (see Ekman \& Friesen, 1969; Laukka, Neiberg, Forsell, Karlsson, \& Elenius, 2011; Scherer, 1989). Also, activations in IFG have consistently been associated with perception of affective prosody (e.g., Leitman et al., 2010; Schirmer \& Kotz, 2006; Wildgruber, Ackermann, Kreifelts, \& Ethofer, 2006). Because inferior frontal regions are recruited for both perception and production of affective prosody, we propose that an explanation of the present findings is that the IFG may be involved in both the monitoring and the modulation of one's own vocal affect expressions. Further research is needed to confirm this proposed role of the IFG, but it is in line with research suggesting that speech production and speech perception are linked in a system where one's own voice production is monitored and modulated by sensory feedback and topdown processes (e.g., Aziz-Zadeh et al., 2010; Zheng, Munhall, \& Johnsrude, 2010).

For our subcortical ROIs, we found negative correlations with expressed vocal affect and activity in the right putamen and hippocampus. Both of these areas have been implicated in previous studies of emotion regulation. Putamen (together with amygdala) has been proposed to be involved in the orienting and perceptual processes that generate emotional states and behaviors (e.g., Ochsner \& Gross, 2005; Phillips et al., 2003). Intriguingly, a recent review of neuroimaging studies of language suggested that the putamen also plays a role in speech production (Price, 2010). The hippocampus, in turn, together with prefrontal 
Table 2 Regions functionally coupled with voxels in the inferior frontal gyrus that covaried with vocally expressed affect, presented in terms of $z$ scores representing local maxima and their associated Talairach coordinates, number of voxels/cluster, and voxelwise $p$ values corrected for multiple comparisons (false discovery rate correction)

\begin{tabular}{|c|c|c|c|c|c|c|}
\hline \multirow[b]{2}{*}{ Region } & \multirow[b]{2}{*}{$z$ score } & \multicolumn{3}{|c|}{ Coordinate } & \multirow{2}{*}{$\begin{array}{l}\text { Voxels in } \\
\text { cluster }\end{array}$} & \multirow{2}{*}{$\begin{array}{l}\text { Voxel } p \\
\text { value }\end{array}$} \\
\hline & & $x$ & $y$ & $z$ & & \\
\hline & \multicolumn{6}{|c|}{ Right IFG Connectivity } \\
\hline \multicolumn{7}{|l|}{$A C C R O I$} \\
\hline Rostral ACC & 4.40 & 6 & 43 & -2 & 876 & .004 \\
\hline \multicolumn{7}{|c|}{ Amygdala/hippocampus ROI } \\
\hline Hippocampus & 3.95 & -36 & -14 & -11 & 59 & .008 \\
\hline Hippocampus & 3.93 & 34 & -31 & -5 & 88 & .008 \\
\hline Amygdala & 3.53 & -18 & 1 & -14 & 15 & .008 \\
\hline \multicolumn{7}{|c|}{ Basal ganglia ROI } \\
\hline Putamen & 4.48 & 34 & 4 & 0 & 183 & .003 \\
\hline Putamen & 4.07 & -22 & 7 & -9 & 317 & .003 \\
\hline \multirow[t]{2}{*}{ Caudate } & 3.52 & 16 & 17 & -6 & 65 & .003 \\
\hline & \multicolumn{6}{|c|}{ Left IFG Connectivity } \\
\hline \multicolumn{7}{|l|}{$A C C R O I$} \\
\hline Dorsal ACC & 4.29 & -4 & 27 & 26 & 1,262 & .004 \\
\hline \multicolumn{7}{|c|}{ Amygdala/hippocampus ROI } \\
\hline Hippocampus & 3.60 & 32 & -31 & -3 & 49 & .035 \\
\hline \multicolumn{7}{|c|}{ Basal ganglia ROI } \\
\hline Putamen & 3.80 & 32 & 8 & 0 & 81 & .017 \\
\hline Pallidum & 3.26 & 20 & -4 & 6 & 20 & .017 \\
\hline Putamen & 3.22 & -22 & 10 & 5 & 51 & .017 \\
\hline
\end{tabular}

areas, has been proposed to be part of a network that is involved in the volitional control of affect (Phillips et al., 2003) and is further strongly associated with inhibition of fear responses during extinction of fear conditioning (Ji \& Maren, 2007). In line with these findings, our results could be indicative of possible roles for the putamen and hippocampus in the modulation of affective prosody.

We also performed an exploratory whole-brain regression analysis between $\mathrm{rCBF}$ and vocally expressed nervousness that revealed activity in several additional brain regions in which $\mathrm{rCBF}$ covaried negatively with affective prosody. Inhibition of actions has previously been suggested to be right lateralized (Garavan, Ross, \& Stein, 1999; Konishi, Nakajima, Uchida, Kikyo, Kameyama, Miyashita, et al., 1999), and the cortical areas showing negative covariation with vocal expression in the exploratory whole-brain analyses were restricted to the right hemisphere. Our data further suggest that the right precuneus and superior temporal cortex may be involved in vocal affect expression. The precuneus has been shown to be involved in self-related mental representations (Cavanna \& Trimble, 2006), and this part of the parietal cortex, together with the superior temporal cortex, has further been found to be involved in the judgment of emotional states of the self and others (Ochsner et al., 2004). Recent studies have implicated areas of the PFC as being functionally connected to the cerebellum (Allen, McColl, Barnard, Ringe, Fleckenstein, Cullum, et al., 2005; Krienen \& Buckner, 2009). In these studies, it is worth noting that the right PFC showed connectivity to the left cerebellum. In line with this contralateral connectivity pattern, whereas all cerebral areas that showed a significant negative covariation with affective prosody in our study were situated in the right hemisphere, the only area in the left hemisphere was found in the left cerebellum. For example, Krienen and Buckner (2009) reported that the right motor cortex showed functional connectivity with the left cerebellum, and intriguingly, we also found a negative covariation between nervousness in voice and the right motor cortex. This suggests that a previously identified cerebellar-motor-prefrontal network might be involved in affective prosody.

We further found that voxels in the IFG that showed a negative correlation to affective prosody were functionally coupled to the frontal ACC. ACC has connections with motor channels (which provide access to motor output systems as well as vocalization), lateral PFC (which provides access to top-down cortical regulation), and also has afferents from the midline thalamus and brain stem that provide a strong modulatory influence of the arousal state (Paus, 2001). We have previously demonstrated that activity in the rostral ACC is negatively correlated with stress-induced cortisol 

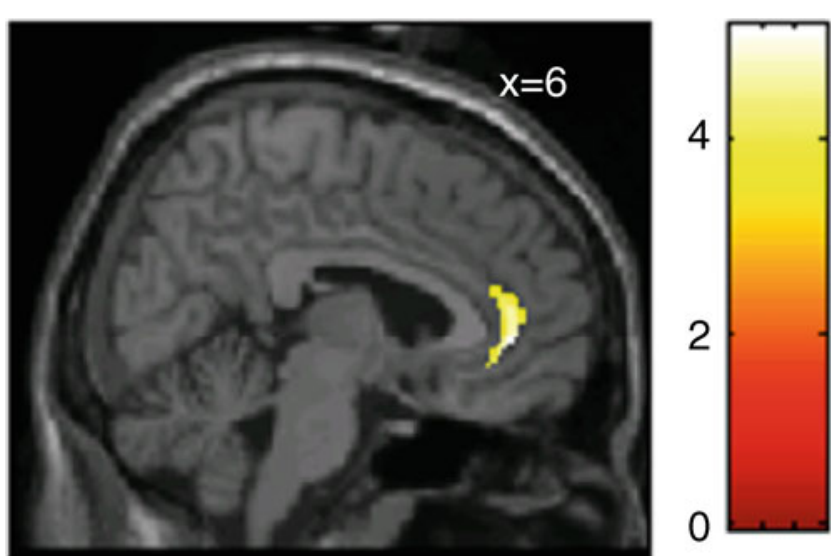

Right IFG vs. ACC

Correlation: $r=0.68$

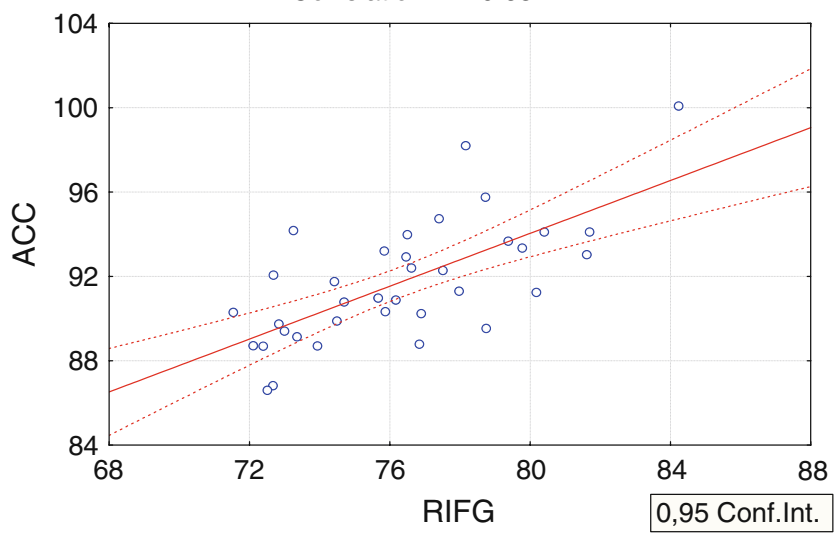

Fig. 2 Functional connectivity analysis map illustrating that voxels in both left and right IFG that showed a negative correlation to vocally expressed affect were functionally coupled to the ACC. The functional images are superimposed on a standard MRI image, and the color bar

response (Åhs, Furmark, Michelgård, Långström, Appel, Wolf, et al., 2006) and positively correlated with vagal control of the heart ( $\AA$ hs, Sollers, Furmark, Fredrikson, \& Thayer, 2009). Thus, the ACC seems to be in a good position to regulate the interaction between cognition, physiological response, and motor control in relation to changes in emotional and motivational states (Bush, Luu, \& Posner, 2000; Paus, 2001). The rostral-ventral regions of the ACC have consistently been linked with emotional functions, whereas dorsal areas subserve more cognitive functions (Bush, Luu, \& Posner, 2000; Rolls, 2005, pp. 181187). In our data, the activations in right ACC overlapped with the "affective division" of the ACC, but the activations in left ACC extended farther into the dorsal areas. Of special interest to affective prosody regulation, the activation in left dorsal ACC corresponds to the presumed speech/vocalization area of the ACC (Paus, Petrides, Evans, \& Meyer, 1993), which has previously been associated with the modulation of prosody during affective speech (Barrett et al., 2004). It has been suggested that lateral PFC, together
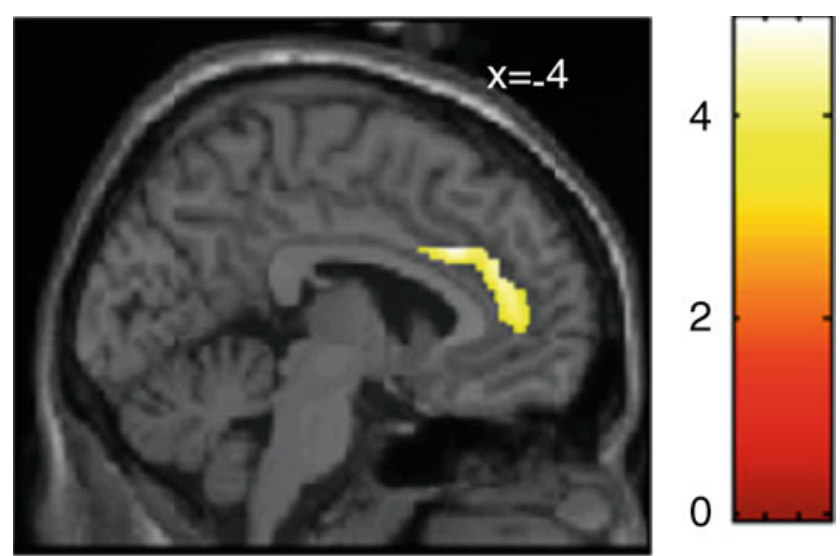

Left IFG vs. ACC

Correlation: $r=0.70$

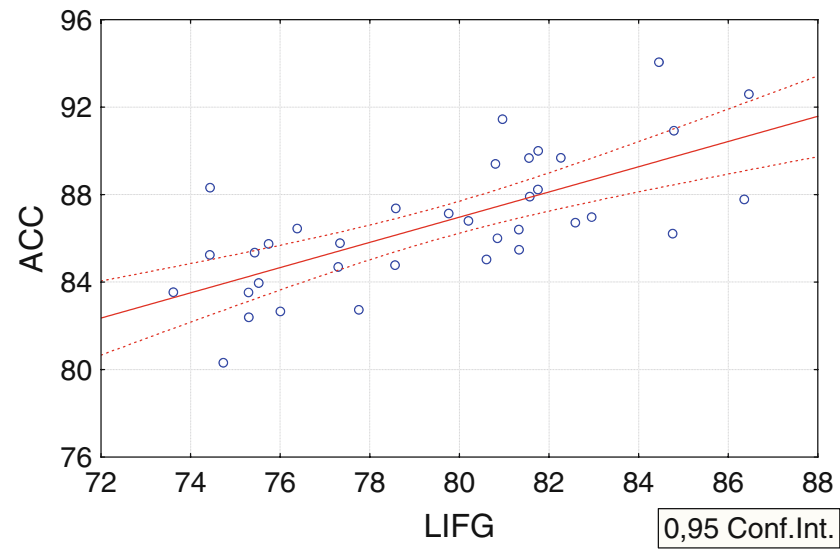

represents $t$ scores. The unit of both axes in the Pearson correlation plot is [ml of regional cerebral blood flow/100 gmin]. Solid line = regression line, dotted lines $=95 \%$ confidence intervals

with ACC, forms part of a top-down appraisal system controlling bottom-up appraisals generated by subcortical regions (e.g., Ochsner \& Gross, 2007). Regarding affective prosody, our results showing IFG-ACC connectivity have tentatively suggested that IFG and ACC may be part of a top-down system associated with the modulation of affective prosody.

Negative functional couplings between vocally expressed affect and activity in the IFG were also revealed for activity in the amygdala, the hippocampus, and the putamen. Amygdala is a key component for the affective appraisal of emotionally and socially relevant information, and is associated with detecting, attending to, and remembering affectively arousing stimuli (Phelps, 2006; Whalen, 1998). The present data suggest functional couplings between the IFG and the amygdala together with the hippocampus when talking about a previous experience in front of an audience. Mirroring this observation, a study on autobiographical memory found increased functional connectivity between the IFG and the amygdala and hippo- 
campus during retrieval (Greenberg et al., 2005). Therefore, it is possible that the connectivity pattern reflects the autobiographical nature of the speech task. We further found functional couplings between the IFG and several areas of the basal ganglia. Because of the involvement of the basal ganglia in speech production (Price, 2010) and of the IFG in both speech production and evaluation of prosody (see, e.g., Aziz-Zadeh et al., 2010; Schirmer \& Kotz, 2006), this coupling might represent a cortico-striatal feedback loop updating motor programs with information regarding how much vocal affect was expressed.

In the present study, we investigated the neural correlates of vocally expressed affect in patients with social phobia, using data obtained from an ongoing treatment study (e.g., Furmark et al., 2005). According to cognitive models of social phobia (e.g., Clarke \& Wells, 1995), individuals with social phobia focus their attention on detailed monitoring of themselves when perceiving social danger, which in turn increases the salience of anxiety-related interoceptive cues (e.g., autonomic arousal). In line with this process, previous research has shown that social anxiety is associated with an excessive use of suppression strategies (e.g., Aldao, NolenHoeksma, \& Schweizer, 2010; Kashdan \& Steger, 2006; Spokas et al., 2009). Suppression of emotional expressions is further known to lead to increased physiological activation but to have little or no effect on self-reported anxiety (Gross \& Levenson, 1997), which is in accord with our results wherein the level of expressed vocal affect was not correlated with the level of self-reported anxiety. Prior studies have further reported that patients with social phobia show increased activation in the amygdala and basal ganglia, together with decreased activity in such cortical regions as PFC and ACC, before and during public-speaking tasks (e.g., Lorberbaum, Kose, Johnson, Arana, Sullivan, Hamner, et al., 2004; Tillfors et al., 2001), and patients with anxiety disorders have shown altered functioning in PFC and ACC during emotion regulation (e.g., Campbell-Sills et al., 2011; Etkin et al., 2010). Taken together, reports such as these suggest that the observed associations between brain regions and expressed vocal affect may possibly be restricted to anxiety disorder patients. Future studies could therefore investigate the differences in affective prosody between socially anxious patients and healthy controls. Such studies would elucidate potential neural mechanisms associated with vocal expression of affect and lead to a better understanding of the neurobiology of social phobia.

Other limitations of the present study include the fact that we did not prompt our patients to use any particular strategy to cope with the stressful speech task. This increased the ecological validity of our study, in the sense that it reflected an unprompted real-life situation, but it also meant that we had no control over what strategies the patients used in order to modulate their nonverbal affect expressions. However, it should be noted that even though all participants were performing the same task in the same anxiety-provoking situation and reported similar levels of anxiety, behavioral measures indicated that some did convey a lot of nervousness through their voice, while others sounded less nervous, which is consistent with some sort of regulation occurring in patients who expressed little, as opposed to much, nervousness. A final limitation is that we did not measure the patients' vocal behavior during a nonemotional speech task, which could have served as an important baseline condition with which to compare the brain activations obtained during emotional speech.

To conclude, the present study was the first to investigate the neural correlates of vocally expressed affect in a real emotion-eliciting situation, and as such it was in many aspects exploratory in character. Our results suggest an involvement of the IFG, the hippocampus, and the putamen in the modulation of affect in voice during an autobiographical speech task. These regions were further functionally connected to one another, which suggests that they may be part of a neural network concerned with the regulation of affective prosody. Given the importance of nonverbal affect expressions for social interaction in both healthy and clinical populations, the neural mechanisms underlying these phenomena deserve more attention.

\section{References}

Åhs, F., Furmark, T., Michelgård, A., Långström, B., Appel, L., Wolf, O. T., et al. (2006). Hypothalamic blood flow correlates positively with stress-induced cortisol levels in subjects with social anxiety disorder. Psychosomatic Medicine, 68, 859-862.

Åhs, F., Sollers, J. J., 3rd, Furmark, T., Fredrikson, M., \& Thayer, J. F. (2009). High-frequency heart rate variability and cortico-striatal activity in men and women with social phobia. NeuroImage, 47, 815-820.

Aitken, P. G. (1981). Cortical control of conditioned and spontaneous vocal behavior in rhesus monkeys. Brain and Language, 13, 171-184.

Aldao, A., Nolen-Hoeksma, S., \& Schweitzer, S. (2010). Emotionregulation strategies across psychopathology: A meta-analytic review. Clinical Psychology Review, 30, 217-237.

Allen, G., McColl, R., Barnard, H., Ringe, W. K., Fleckenstein, J., \& Cullum, C. M. (2005). Magnetic resonance imaging of cerebellar-prefrontal and cerebellar-parietal functional connectivity. NeuroImage, 28, 39-48.

Ambady, N., \& Rosenthal, R. (1992). Thin slices of expressive behavior as predictors of interpersonal consequences: A metaanalysis. Psychological Bulletin, 111, 256-274.

Aziz-Zadeh, L., Sheng, T., \& Gheytanchi, A. (2010). Common premotor regions for the perception and production of prosody and correlations with empathy and prosodic ability. PLOS ONE, 5, e8759. doi:10.1371/journal.pone.0008759.

Banks, S. J., Eddy, K. T., Angstadt, M., Nathan, P. J., \& Phan, K. L. (2007). Amygdala-frontal connectivity during emotion regulation. Social Cognitive and Affective Neuroscience, 2, $303-312$. 
Barrett, J., Pike, B., \& Paus, T. (2004). The role of the anterior cingulate cortex in pitch variation during sad affect. European Journal of Neuroscience, 19, 458-464.

Baum, S. R., \& Pell, M. D. (1999). The neural bases of prosody: Insights from lesion studies and neuroimaging. Aphasiology, 13, 581-608.

Boersma, P., \& Weenink, D. (2007). Praat: Doing phonetics by computer [Computer program]. Retrieved from www.praat.org.

Bush, G., Luu, P., \& Posner, M. I. (2000). Cognitive and emotional influences in anterior cingulate cortex. Trends in Cognitive Sciences, 4, 215-222.

Campbell-Sills, L., Barlow, D. H., Brown, T. A., \& Hofmann, S. G. (2006). Acceptability and suppression of negative emotion in anxiety and mood disorders. Emotion, 6, 587-595.

Campbell-Sills, L., Simmons, A. N., Lovero, K. L., Rochlin, A. A., Paulus, M. P., \& Stein, M. B. (2011). Functioning of neural systems supporting emotion regulation in anxiety-prone individuals. NeuroImage, 54, 689-696.

Cavanna, A. E., \& Trimble, M. R. (2006). The precuneus: A review of its functional anatomy and behavioral correlates. Brain, 129, 564-583.

Clarke, D. M., \& Wells, A. (1995). A cognitive model of social phobia. In R. G. Heimberg, M. R. Liebowitz, D. A. Hope, \& F. R. Schneier (Eds.), Social phobia: Diagnosis, assessment and treatment (pp. 69-93). New York: Guilford Press.

Cowie, R., \& Cornelius, R. R. (2003). Describing the emotional states that are expressed in speech. Speech Communication, $40,5-32$.

Davidson, R. J. (2002). Anxiety and affective style: Role of prefrontal cortex and amygdala. Biological Psychiatry, 51, 68-80.

Davidson, R. J., Fox, A., \& Kalin, N. H. (2007). Neural bases of emotion regulation in nonhuman primates and humans. In J. J. Gross (Ed.), Handbook of emotion regulation (pp. 47-68). New York: Guilford Press.

Ekman, P., \& Friesen, W. V. (1969). The repertoire of nonverbal behavior: Categories, origins, usage, and coding. Semiotica, 1, 49-98.

Engel, K., Bandelow, B., Gruber, O., \& Wedekind, D. (2009). Neuroimaging in anxiety disorders. Journal of Neural Transmission, 116, 703-716.

Ethofer, T., Van De Ville, D., Scherer, K., \& Vuilleumier, P. (2010). Decoding of emotional information in voice-sensitive cortices. Current Biology, 19, 1028-1033.

Etkin, A., \& Wager, T. D. (2007). Functional neuroimaging of anxiety: A meta-analysis of emotional processing in PTSD, social anxiety disorder, and specific phobia. American Journal of Psychiatry, 164, 1476-1488

Etkin, A., Prater, K. E., Hoeft, F., Menon, V., \& Schatzberg, A. F. (2010). Failure of anterior cingulate activation and connectivity with the amygdala during implicit regulation of emotional processing in generalized anxiety disorder. American Journal of Psychiatry, 167, 545-554.

First, M. B., Gibbon, M., Spitzer, R. L., \& Williams, J. B. W. (1998). Structured Clinical Interview for DSM-IV Axis I Disorders (SCID-I): Interview protocol [Swedish]. Danderyd, Sweden: Pilgrim. [Swedish translation by J. Herlofson].

Freitas-Ferrari, M. C., Hallak, J. E. C., Trzesniak, C., Filho, A. S., Machado-de-Sousa, J. P., Chagas, M. H. N., et al. (2010). Neuroimaging in social anxiety disorder: A systematic review of the literature. Progress in Neuro-Psychopharmacology and Biological Psychiatry, 34, 565-580.

Friston, K. J., Holmes, A. P., Worsley, K. J., Poline, J.-P., Frith, C. D., \& Frackowiak, R. S. J. (1994). Statistical parametric maps in functional imaging: A general linear approach. Human Brain Mapping, 2, 189-210.

Furmark, T., Appel, L., Michelgård, Å., Wahlstedt, K., Åhs, F., Zancan, S., et al. (2005). Cerebral blood flow changes after treatment of social phobia with the neurokinin-1 antagonist GR205171, citalopram, or placebo. Biological Psychiatry, 58, 132-142.

Furmark, T., Tillfors, M., Everz, P.-O., Marteinsdottir, I., Gefvert, O., \& Fredrikson, M. (1999). Social phobia in the general population: Prevalence and sociodemographic profile. Social Psychiatry and Psychiatric Epidemiology, 34, 416-424.

Furmark, T., Tillfors, M., Marteinsdottir, I., Fischer, H., Pissiota, A., Långström, B., et al. (2002). Common changes in cerebral blood flow in patients with social phobia treated with citalopram or cognitive-behavioral therapy. Archives of General Psychiatry, $59,425-433$.

Garavan, H., Ross, T. J., \& Stein, E. A. (1999). Right hemispheric dominance of inhibitory control: An event-related functional MRI study. Proceedings of the National Academy of Sciences, 96, 8301-8306.

Genovese, C. R., Lazar, N. A., \& Nichols, T. (2002). Thresholding of statistical maps in functional neuroimaging using the false discovery rate. NeuroImage, $15,870-878$.

Goldin, P. R., Manber, T., Hakimi, S., Canli, T., \& Gross, J. J. (2009). Neural bases of social anxiety disorder: Emotional reactivity and cognitive regulation during social and physical threat. Archives of General Psychiatry, 66, 170-180.

Goldin, P. R., McRae, K., Ramel, W., \& Gross, J. J. (2008). The neural bases of emotion regulation: Reappraisal and suppression of negative emotion. Biological Psychiatry, 63, 577-586.

Greenberg, D. L., Rice, H. J., Cooper, J. J., Cabeza, R., Rubin, D. C., \& LaBar, K. S. (2005). Co-activation of the amygdala, hippocampus and inferior frontal gyrus during autobiographical memory retrieval. Neuropsychologia, 43, 659-674.

Gross, J. J. (1998). The emerging field of emotion regulation: An integrative review. Review of General Psychology, 2, 271-299.

Gross, J. J., \& Levenson, R. W. (1997). Hiding feelings: The acute effects of inhibiting negative and positive emotion. Journal of Abnormal Psychology, 106, 95-103.

Hagemann, T., Levenson, R. W., \& Gross, J. J. (2006). Expressive suppression during an acoustic startle. Psychophysiology, 43, 104-112.

Ji, J., \& Maren, S. (2007). Hippocampal involvement in contextual modulation of fear extinction. Hippocampus, 17, 749-758.

Johnstone, T., van Reekum, C. M., Urry, H. L., Kalin, N. H., \& Davidson, R. J. (2007). Failure to regulate: Counterproductive recruitment of top-down prefrontal-subcortical circuitry in major depression. Journal of Neuroscience, 27, 8877-8884.

Jürgens, U. (2002). Neural pathways underlying vocal control. Neuroscience and Biobehavioral Reviews, 26, 235-258.

Jürgens, U. (2009). The neural control of vocalization in mammals: A review. Journal of Voice, 23, 1-10.

Jürgens, U., \& von Cramon, D. (1982). On the role of the anterior cingulate cortex in phonation: A case report. Brain and Language, 15, 234-248.

Juslin, P. N., \& Laukka, P. (2003). Communication of emotion in vocal expression and music performance: Different channels, same code? Psychological Bulletin, 129, 770-814.

Kashdan, T. B., \& Steger, M. F. (2006). Expanding the topography of social anxiety: An experience-sampling assessment of positive emotions, positive events and emotion suppression. Psychological Science, 17, 120-128.

Kessler, R. C., Stein, M. B., \& Berglund, P. (1998). Social phobia subtypes in the National Comorbidity Survey. American Journal of Psychiatry, 155, 613-619.

Kober, H., Barrett, L. F., Joseph, J., Bliss-Moreau, E., Lindquist, K., \& Wager, T. D. (2008). Functional grouping and cortical-subcortical interactions in emotion: A meta-analysis of neuroimaging studies. NeuroImage, 42, 998-1031.

Konishi, S., Nakajima, K., Uchida, I., Kikyo, H., Kameyama, M., \& Miyashita, Y. (1999). Common inhibitory mechanism in human 
inferior prefrontal cortex revealed by event-related functional MRI. Brain, 122, 981-991.

Koole, S. L. (2009). The psychology of emotion regulation: An integrative review. Cognition and Emotion, 23, 4-41.

Krienen, F. M., \& Buckner, R. L. (2009). Segregated fronto-cerebellar circuits revealed by intrinsic functional connectivity. Cerebral Cortex, 19, 2485-2497.

Kring, A. M., \& Bachorowski, J.-A. (1999). Emotions and psychopathology. Cognition and Emotion, 13, 575-599.

Lancaster, J. L., Woldorff, M. G., Parsons, L. M., Liotti, M., Freitas, C. S., Rainey, L., et al. (2000). Automated Talairach atlas labels for functional brain mapping. Human Brain Mapping, 10, 120-131.

Lane, R. D., \& McRae, K. (2004). Neural substrates of conscious emotional experience: A cognitive-neuroscientific perspective. In M. Beauregard (Ed.), Consciousness, emotional self-regulation and the brain (pp. 87-122). Amsterdam: Benjamins.

Laukka, P. (2008). Research on vocal expression of emotion: State of the art and future directions. In K. Izdebski (Ed.), Emotions in the human voice (Foundations, Vol. 1, pp. 153-169). San Diego: Plural Publishing.

Laukka, P., Linnman, C., Åhs, F., Pissiota, A., Frans, Ö., Faria, V., et al. (2008). In a nervous voice: Acoustic analysis and perception of anxiety in social phobics' speech. Journal of Nonverbal Behavior, 32, 195-214.

Laukka, P., Neiberg, D., Forsell, M., Karlsson, I., \& Elenius, K. (2011). Expression of affect in spontaneous speech: Acoustic correlates, perception, and automatic detection of irritation and resignation. Computer Speech and Language, 25, 84-104.

Lee, T. W., Josephs, O., Dolan, R. J., \& Critchley, H. D. (2006). Imitating expressions: Emotion-specific neural substrates in facial mimicry. Social Cognitive and Affective Neuroscience, 1, 122-135.

Leitman, D. I., Wolf, D. H., Ragland, J. D., Laukka, P., Loughead, J., Valdez, J. N., et al. (2010). "It's not what you say, but how you say it": A reciprocal temporo-frontal network for affective prosody. Frontiers in Human Neuroscience, 4, Art. 19. doi:10.3389/fnhum.2010.00019.

Lieberman, M. D. (2007). Social cognitive neuroscience: A review of core processes. Annual Review of Psychology, 58, 259-289.

Lorberbaum, J. P., Kose, S., Johnson, M. R., Arana, G. W., Sullivan, L. K., Hamner, M. B., et al. (2004). Neural correlates of speech anticipatory anxiety in generalized social phobia. NeuroReport, $15,2701-2705$.

MacLean, P. D., \& Newman, J. D. (1988). Role of midline frontolimbic cortex in production of the isolation call of squirrel monkeys. Brain Research, 450, 111-123.

Maldjian, J. A., Laurienti, P. J., Kraft, R. A., \& Burdette, J. H. (2003). An automated method for neuroanatomic and cytoarchitectonic atlas-based interrogation of fMRI data sets. NeuroImage, 19, $1233-1239$

Nichols, T., \& Hayasaka, S. (2003). Controlling the familywise error rate in functional neuroimaging: A comparative review. Statistical Methods in Medical Research, 12, 419-446.

Ochsner, K. N., \& Gross, J. J. (2005). The cognitive control of emotion. Trends in Cognitive Sciences, 9, 242-249.

Ochsner, K. N., \& Gross, J. J. (2007). The neural architecture of emotion regulation. In J. J. Gross (Ed.), Handbook of emotion regulation (pp. 87-109). New York: Guilford Press.

Ochsner, K. N., Bunge, S. A., Gross, J. J., \& Gabrieli, J. D. E. (2002). Rethinking feelings: An fMRI study of the cognitive regulation of emotion. Journal of Cognitive Neuroscience, 14, $1215-1229$.

Ochsner, K. N., Knierim, K., Ludlow, D. H., Hanelin, J., Ramachandran, T., Glover, G., et al. (2004). Reflecting upon feelings: An fMRI study of neural systems supporting the attribution of emotion to self and other. Journal of Cognitive Neuroscience, 16, 1746-1772.
Paus, T. (2001). Primate anterior cingulate cortex: Where motor control, drive and cognition interface. Nature Reviews Neuroscience, 2, $417-424$

Paus, T., Petrides, M., Evans, A. C., \& Meyer, E. (1993). Role of the human anterior cingulate cortex in the control of oculomotor, manual, and speech responses: A positron emission tomography study. Journal of Neurophysiology, 70, 453-469.

Phelps, E. A. (2006). Emotion and cognition: Insights from studies of the human amygdala. Annual Review of Psychology, 57, 27-53.

Phillips, M. L., Drevets, W. C., Rauch, S. L., \& Lane, R. (2003). Neurobiology of emotion perception I: The neural basis of normal emotion perception. Biological Psychiatry, 54, 504-514.

Phillips, M. L., Ladouceur, C. D., \& Drevets, W. C. (2008). A neural model of voluntary and automatic emotion regulation: Implications for understanding the pathophysiology and neurodevelopment of bipolar disorder. Molecular Psychiatry, 13, 833-857.

Price, C. J. (2010). The anatomy of language: A review of $100 \mathrm{fMRI}$ studies published in 2009. Annals of the New York Academy of Sciences, 1191, 62-88.

Quirk, G. J., \& Beer, J. S. (2006). Prefrontal involvement in the regulation of emotion: Convergence of rat and human studies. Current Opinion in Neurobiology, 16, 723-727.

Rolls, E. T. (2005). Emotion explained. New York: Oxford University Press.

Ross, E. (1981). The aprosodias: Functional-anatomic organization of the affective components of language in the right hemisphere. Archives of Neurology, 38, 561-569.

Russell, J. A., Bachorowski, J.-A., \& Fernández-Dols, J.-M. (2003). Facial and vocal expressions of emotion. Annual Review of Psychology, 54, 329-349.

Scherer, K. R. (1989). Vocal correlates of emotional arousal and affective disturbance. In H. Wagner \& A. Manstead (Eds.), Handbook of social psychophysiology (pp. 165-197). New York: Wiley.

Scherer, K. R. (2000). Psychological models of emotion. In J. Borod (Ed.), The neuropsychology of emotion (pp. 137-162). New York: Oxford University Press.

Scherer, K. R. (2003). Vocal communication of emotion: A review of research paradigms. Speech Communication, 40, 227-256.

Scherer, K. R., Koivumaki, J., \& Rosenthal, R. (1972). Minimal cues in the vocal communication of affect: Judging emotions from content-masked speech. Journal of Psycholinguistic Research, 1, 269-285.

Schirmer, A., \& Kotz, S. A. (2006). Beyond the right hemisphere: Brain mechanisms mediating vocal emotional processing. Trends in Cognitive Sciences, 10, 24-30.

Spielberger, C. D., Gorsuch, R. L., Lushene, R. E., Vagg, P. R., \& Jacobs, G. A. (1983). Manual for the State-Trait Anxiety Inventory. Palo Alto, CA: Consulting Psychologists Press.

Spokas, M., Luterek, J. A., \& Heimberg, R. G. (2009). Social anxiety and emotional suppression: The mediating role of beliefs. Journal of Behavior Therapy and Experimental Psychiatry, 40, 283-291.

Talairach, J., \& Tournoux, P. (1988). Co-planar stereotaxic atlas of the human brain: 3-dimensional proportional system. An approach to cerebral imaging. Stuttgart: Thieme.

Tillfors, M., Furmark, T., Marteinsdottir, I., Fischer, H., Pissiota, A., Långström, B., et al. (2001). Cerebral blood flow in subjects with social phobia during stressful speaking tasks: A PET study. American Journal of Psychiatry, 158, 1220-1226.

Urry, H. A., van Reekum, C. M., Johnstone, T., Kalin, N. H., Thurow, M. E., Schaefer, H. S., et al. (2006). Amygdala and ventromedial prefrontal cortex are inversely coupled during regulation of negative affect and predict the diurnal pattern of cortisol secretion among older adults. Journal of Neuroscience, 26, 4415-4425.

van Bezooijen, R., \& Boves, L. (1986). The effects of low-pass filtering and random splicing on the perception of speech. Journal of Psycholinguistic Research, 15, 403-417. 
von Cramon, D., \& Jürgens, U. (1983). The anterior cingulate cortex and the phonatory control in monkey and man. Neuroscience and Biobehavioral Reviews, 7, 423-425.

Wager, T. D., Davidson, M. L., Hughes, B. L., Lindquist, M. L., \& Ochsner, K. N. (2008). Prefrontal-subcortical pathways mediating successful emotion regulation. Neuron, 59, 1037-1050.

Whalen, P. J. (1998). Fear, vigilance, and ambiguity: Initial neuroimaging studies of the human amygdala. Current Directions in Psychological Science, 7, 177-188.

Wildgruber, D., Ackermann, H., Kreifelts, B., \& Ethofer, T. (2006). Cerebral processing of linguistic and emotional prosody: fMRI studies. Progress in Brain Research, 156, 249-268.
Zheng, Z. Z., Munhall, K. G., \& Johnsrude, I. S. (2010). Functional overlap between regions involved in speech perception and in monitoring one's own voice during speech production. Journal of Cognitive Neuroscience, 22, 1770-1781.

\section{Author Note}

This study was supported by the Swedish Research Council, the Swedish Council for Working Life and Social Research, the Bank of Sweden Tercentenary Foundation, and the Brain Foundation. 